\title{
A Classification of Industrial Symbiosis Networks: A Focus on Materials and Energy Recovery
}

\author{
Vito Albino, Achille Claudio Garavelli, and Vincenzo Alessio Romano \\ DMMM, Politecnico di Bari, \\ Viale Japigia 182, 70126 Bari, Italy \\ \{v.albino, c.garavelli, va.romano\}@poliba.it
}

\begin{abstract}
Industrial symbiosis (IS) has gained more attention in the production economics as the pressure on companies increases for the reduction of waste emissions as well as of primary resources consumption. At present, as the number of IS initiatives increases, many issues about its boundaries, such as the entities involved and the resources shared/exchanged, still continue to remain open. A common classification of IS networks does still not exist.

Input-output matching is the most significant tool to analyze IS. To this aim, we adopt an input-output approach for defining an IS network, and for proposing a classification model at the technical level (i.e. physical flow type, number of production chains involved, and network structure).

The proposed approach is applied to several existing IS networks to describe as it works. This classification framework can be useful for companies to set strategies and for local government to plan policies.
\end{abstract}

Keywords: sustainable production, industrial symbiosis, classification.

\section{Introduction}

Nowadays, problems related to the natural resources consumption, energy usage, Greenhouse Gas (GHG) emissions, and fossil fuel utilization are becoming more and more crucial and relevant. Therefore, there is a need to give further impetus to efficient and eco-innovative production processes, to reduce dependency on raw materials, and to encourage optimal resource use and recycling (UNEP, 2009). Industrial symbiosis (IS) has been considered as one of the most effective solutions to reduce the impact of waste emissions and of primary input consumption moving towards sustainable production models. The Organization for Economic Co-operation and Development has recently identified IS as a tool for systemic innovation vital for future green growth (OECD, 2010). Furthermore, within the Resource Efficiency Flagship Initiative, IS is indicated as a tool that all member states should exploit to help companies work together to make the best use of the waste and by-products they produce (EU, 2011).

Industrial symbiosis has emerged as a sub-field of industrial ecology. "Industrial ecology is the science that provides the conceptual tools to analyze and optimize the flow of energy and materials in production systems" (The Interagency Workgroup on 
Industrial Ecology, Material and Energy Flows, 1998). In particular, IS operates at the inter-firm level (Chertow, 2000). However, despite over twenty years of industrial ecology, IS phenomenon is still not clearly defined.

IS has been inspired by the concept of "industrial ecosystem" in which "the consumption of energy and materials is optimized and the effluents of one process ... serve as the raw material for another process" (Frosch and Gallopoulos, 1989). More recently, IS was widely known as "engaging traditionally separate industries in a collective approach to competitive advantage involving physical exchange of materials, energy, water and/or by-products" (Chertow, 2000), whose key success factors are "collaboration and the synergistic possibilities offered by geographic proximity". These keys for the success of IS differ according to the case- and location-specificity of IS, and they have been largely discussed within the recent literature, often achieving not fully agreed results.

Additionally, through the years, the IS network concept has been extended not only to all types of physical and unphysical resources that could be intentionally shared or exchanged (by-products, utilities, and ancillary services) (Chertow, 2007), but also to all the other firm resources such as, for example, knowledge and social processes.

Therefore, IS has been approached from various perspectives (e.g. social, economic, environmental, spatial, organizational, technical). Nevertheless, with the increasing number of IS projects, many issues about IS boundaries, such as the entities involved (firms, organizations, industries, facilities) and the resources shared/exchanged (materials, energy, water, information, expertise), still continue to remain open.

With this regard, focusing on materials and energy physical flows only, and adopting an input-output (I-O) approach, we define an IS network as a set of one or more production chains utilizing their waste materials and residual energy in substitution of primary resources. In particular, we propose a technical classification model of IS networks based on the physical flow type involved, and the production chains sending and receiving it. The study aims at proposing a basic categorization of IS networks, a classification tool, which could be a common ground for successive structural network analyses.

In the next section a specific review of the literature is devoted to the materials and energy flows in IS networks. In the third section the classification model for IS networks is proposed. In the fourth section several case examples are provided. Finally, main conclusions are reported in the last section.

\section{Materials and Energy in IS Networks}

From a technical point of view, a production chain can be considered as an inputoutput system (Storper and Harrison, 1992) that describes the product flows existing among production processes. Input-output systems may involve many production processes, depending on a specific division and classification of production in processes. In general, the chain may contain the extraction of raw materials, manufacturing, distribution and use of goods. Referring to the environmental 
sustainability, a sustainable production chain means favouring, for a given output, processes with less use of natural resources (energy and materials) and production of wastes. From the supply perspective, extended supply chains include the reduction and elimination of by-products through cleaner process technologies and lean production techniques, extending chain boundaries as far as to include the source and the destination of all the physical flows used and produced at each chain stage (Albino et al., 2002; 2003). As the opportunity offered by the efficient use of materials (recycling, etc.) and energy stresses the relevance of network of actors, cooperation between actors becomes crucial.

Cooperation can be considered also in terms of industrial ecology. Industrial symbiosis is the main approach introduced by the industrial ecology to explain how industrial ecosystem (Frosch and Gallopoulos, 1989) may work. Two industrial actors operate in IS when they exchange materials and energy to reduce costs, create value, and improve the environment. Although the analysis of the role and level of cooperation between actors of production chains in permitting environmental and economic benefits needs to be explored further, ownership issues are not faced in our study. We aim at describing symbiotic relationships among industrial actors in terms of materials and energy flows only.

Assuming that symbiotic transactions are waste exchanges, the first technical classification of IS networks is proposed by Chertow (2000), identifying ISs i) within a firm, facility or organization, ii) among firms collocated in a defined eco-industrial park, iii) among local firms that are not co-located, and iv) among firms organized "virtually" in a broader region. However, this categorization seems to be in contrast with the Chertow's (2000) IS definition, which indentifies the no well-quantified geographic proximity as the IS key driver. Lombardi and Laybourn (2012) redefine IS as "engaging diverse organizations in a network ... to yield mutually profitable transactions for novel sourcing of required inputs, and value added destinations for non-product outputs". As the decennary National Industrial Symbiosis Programme (NISP) experience in the United Kingdom demonstrates, the localization of the entities exchanging resources, although geographic proximity is often associated with IS, is not essential for the success of IS networks (Lombardi and Laybourn, 2012). Distance between entities can be considered as a business-as-usual factor.

In this scenario, input-output matching is the most significant tool to analyze IS (Chertow, 2000). In input-output matching, firms systematically collect input and output data of companies and try to create links among partners. Recently, Bossilkov et al. (2005) proposed a technical classification of existing IS networks in three dimensions: i) type of resource exchange (water, energy, process or non-process waste, utility sharing); ii) type of processing involved (direct use or reuse, energy recovery or alternative fuels, material recovery, etc.); and iii) type of synergy (bi or multi-lateral, etc.). However, this classification continues to leave ambiguity about IS boundaries. Exchanged/shared resources and involved actors, which should characterize IS networks and distinguish them from other types of resource synergy, are still not clearly defined. As Golev and Corder (2012) affirm, a common classification for IS networks does still not exist. 


\section{A Classification Model for IS Networks}

Adopting an input-output (I-O) approach, we define an IS network as a set of one or more production chains utilizing their waste materials and residual energy in substitution of primary resources. Thus, we propose a three-dimensional structural classification model for IS networks by tracking material and energy flows among symbiotic chains. This classification refers to an elementary IS network, i.e. a physical flow type (i.e. one waste-one primary input) shared by one or more actors (i.e. production chains). Starting from this basic categorization, we may represent and classify a more complex IS network as a composition of elementary IS networks.

\subsection{Physical Flow Type}

Let us consider an IS network constituted by one or more production chains.

Let us assume that the waste $l$ output flow is the only waste material flow produced, and the primary input type $k$ the only one required by the IS network. Assuming that the waste $l$ can be used as primary input $k$, we may distinguish the i) material-material, and ii) material-energy flow types. In the first case, the waste $l$ can be directly used in production processes without further transformation as material input, or recycled obtaining the primary input required by the IS network. In the second case, the waste $l$ can be used to produce energy (e.g. alternative fuels).

Now let us suppose that the waste $l$ output is an energy flow, and the primary energy input $k$ the only required by the IS network. Assuming that the waste $l$ can be used as primary input $k$, we may identify the iii) energy-energy flow type, i.e. an energy cascade, such as heat recovery.

\subsection{Internal and External IS}

Let us consider an IS network constituted by one production chain, $\alpha$. Let us assume that the chain $\alpha$ only produces the waste type $l$, and only requires the primary input type $k$. Assuming that the waste type $l$ can be used as primary input $k$, a form of internal IS may occur if the chain $\alpha$ uses the waste $l$ as primary input $k$.

Let us consider now an IS network constituted by two production chains, $\alpha$ and $\beta$, where $\alpha \neq \beta$. Let us assume that the chain $\alpha$ only produces the waste type $l$, and no primary input is required, and the chain $\beta$ only requires the primary input type $k$, and no waste is produced. Assuming that the waste type $l$ can be used as primary input $k$, a form of external IS may occur if the chain $\beta$ uses the waste $l$ as primary input $k$.

\subsection{IS Network Structure}

Let us consider an IS network constituted by two or more production chains. Let us assume that the waste type $l$ is only produced, and the primary input $k$ only required by the IS network. Finally, let us assume that the waste $l$ can be used as primary input $k$. For each physical flow type, depending on the number of production chains sending 
and receiving it, we may identify the following configurations: i) one-to-one, ii) oneto- $n$, iii) $n$-to-one, and iv) $m$-to- $n$, where $m, n \geq 2$.

In the first case, let us suppose that the IS network is composed by two production chains, $\alpha$ and $\beta$. Moreover, let us assume that the chain $\alpha$ only produces the waste $l$, and no primary input is required, and the chain $\beta$ only requires the primary input type $k$, and no waste is produced. The "one-to-one" configuration occurs if the chain $\beta$ uses the waste $l$ as primary input $k$.

In the second case, let us suppose that the IS network is composed by three production chains, $\alpha, \beta$, and $\gamma$. Furthermore, let us assume that the chain $\alpha$ only produces the waste $l$, and no primary input is required, and the chains $\beta$ and $\gamma$ only require the primary input $k$, and no waste is produced. The "one-to- $n$ " configuration occurs if the chains $\beta$ and $\gamma$ use the waste $l$ as primary input $k$.

In the third case, let us continue to suppose that the IS network is composed by three production chains, $\alpha, \beta$, and $\gamma$. Let us assume that chains $\beta$ and $\gamma$ only produce the waste $l$, and do not require any primary input, and the chain $\alpha$ only requires the primary input type $k$, and no waste is produced. The " $n$-to-one" configuration occurs if the chains $\alpha$ uses the waste $l$ as primary input $k$.

In the fourth case, let us suppose that the IS network is composed by four production chains, $\alpha, \beta, \gamma$, and $\delta$. Let us assume that chains $\alpha$ and $\beta$ only produce the waste $l$, and do not require any primary input, and chains $\gamma$ and $\delta$ only require the primary input $k$, and do not produce any waste. The " $m$-to- $n$ " configuration occurs if chains $\gamma$ and $\delta$ use the waste type $l$ as primary input $k$.

\section{Case Examples}

As we have anticipated at the beginning of the previous section, a complex IS network may be a chain or a network of elementary IS networks. In this section, we describe several case examples of actual IS networks that better fit our classification model, based on a single one waste-one input physical flow type.

Yang and Feng (2008) analyze the Nanning Sugar Co., Ltd. case, which is one of the most successfully examples of a new form of IS emerged in China, inspired by the "circular economy" philosophy. Black liquor generated during the alkaline pulping process passes through a recovery process. On the one hand, alkali recovered is reintroduced into the pulping process; on the other hand, lime sludge is used in substitution of limestone to produce cement. In this case, if we consider each physical flow type as a one waste-one input flow, we have two "one-to-one" configurations, one internal, and one external. However, if we categorize a physical flow type only based on the waste output flow, independently from the obtainable input types, this is an example of a "one-to- $n$ " configuration, characterized by a material-material flow, and internal-external IS.

Wolf (2007) investigates the Swedish forest industry in the Östergötland region. Three actors are identified: the pulp mill, the saw mill, and the pellet production plant. Sawdust and wood chip by-products from the saw mill are delivered to the pulp mill to be used as biofuel. Thus, the pulp mill supplies steam and electricity to the saw 
mill, and to the pellet chain. As shown, this is a "one-to- $n$ " IS network, both internal and external, with a material-energy flow, where the pulp mill operates as a conversion process.

Research Triangle Institute (1996) has developed a prototype eco-industrial park for the Brownsville (Texas, U.S.) / Matamoros (Mexico) industrial area, identifying opportunities for symbiotic exchanges among local existing companies. In particular, we focus on scrap plastic re-use. The proposed prototype suggests an IS network composed by a plastic recycler, and three other entities sending their plastic scrap: a textile company, and auto and discrete parts manufacturers. Therefore, plastic may be purchased in the form of plastic pellets by the discrete parts manufacturer, after being processed. This is a " $n$-to-one" configuration, with a material-material flow type, and internal-external symbiosis.

Chertow and Miyata (2011) investigate some IS initiatives of a cluster of companies located in the Campbell Industrial Park in Honolulu, HI, on the island of Oahu. In particular, three organizations bring their used activated carbon to AES Hawaii cogeneration plant as alternative fuels: the Honolulu Board of Water Supply (BWS), Tesoro (i.e. an oil refining company), and the Kalaeloa Cogeneration Plant (i.e. an oil-fired power plant). Both BWS and Tesoro pay AES Hawaii to take and burn the used carbon (McCann, 2005). We may categorize this case as a " $n$-to-one" configuration, with a material-energy flow type, and external IS only.

Mirata and Emtairah (2005) study the case of the industrial town of Landskrona, located in south-western Sweden. Several sectors are included such as chemicals, waste management, metals recycling, and public infrastructures. However, if we consider the local community as a third actor, a heat cascading initiative involves other two entities: steel dust and lead battery recycling companies. Heat from lead acid battery and steel dust recyclers is used for district heating by the local community. A " $n$-to-one"/energy-energy/external IS occurs by tracking the heat flow.

Focusing on utility synergies, Van Beers et al. (2007) investigate the Kwinana Industrial Area, dominated by heavy process industries, and located in Western Australia. In particular, a " $m$-to- $n$ "/material-material/internal-external IS occurs by tracking the wastewater flow. In fact, the cogeneration (i.e. Verve Energy) and the chlor-alkali (i.e. Nufarm Coogee) plants send their wastewater to the treatment plant of Tiwest, a company producing titanium dioxide. So, the potable and demineralized water returns to the plants that generate the wastewater flow.

Korhonen (2001) analyzes the regional energy supply system of the Jyvaskyla region in Finland. The Rauhalahti CHP plant, which is a publicly owned body, receives waste wood from a plywood mill, located in the Saynatsalo suburb, and external fuel, such as peat, saw-mill and forestry waste from other actors. Thus, it produces electricity for all households, services and industry in the city, the energy produced by waste is used for district heating, and industrial steam is sent to the Kangas paper mill. This is an example of a " $m$-to- $n$ " configuration, with a materialenergy flow type, and external symbiosis. 


\section{Conclusions}

Industrial symbiosis (IS) is emerging as one of the most effective tools to mitigate environmental impact of industrial activities, by reducing both primary resources consumption and waste emissions. However, no common classification model for IS networks exists (Golev and Corder, 2012). In this paper, adopting an input-output approach, we have defined an elementary IS network based on the single physical flow type (one waste-one primary input) shared among production chains. Furthermore, we have distinguished between internal and external forms of IS. The former occurs when the waste output flow is sent and received by the same chain, the latter when the chain sending and the one receiving it are not the same. These two conditions can co-exist according to the structure of the network. Four possible configurations have been found depending on the number of production chains sending and receiving the physical flow type.

Several case examples of existing IS networks have been also provided. Focusing only on one specific physical flow type (one waste-one primary input), we have classified each case.

The proposed classification model for IS networks does not aim at being an exhaustive categorizing tool, but a basic categorization at the technical level useful for successive network analyses. It can support policy design as network identification provides guidelines for incentives in terms of transportation and environmental legacy issues, as well as of type and size of sectors to be involved in the IS network.

Acknowledgements. Financial support provided by the POR Puglia 2000-2006 (CIP_PE007 - "Metodologie innovative per lo sviluppo di mercati organizzati di servizi logistici”, CIP_PS092 _"Produzione Distribuita come Sistema Innovativo DIPIS and CIP_PE109 - "Development of an innovative model for self-evaluation of the degree of corporate social responsibility") is gratefully acknowledged.

\section{References}

Albino, V., Dietzenbacher, E., Kühtz, S.: Analyzing Material and Energy Flows in an Industrial District using an Enterprise Input-Output Model. Economic Systems Research 15, 457-480 (2003)

Albino, V., Izzo, C., Kühtz, S.: Input-output models for the analysis of a local/global supply chain. Int. J. Product. Econ. 78, 119-131 (2002)

Bossilkov, A., van Berkel, R., Corder, G.: Regional Synergies for Sustainable Resource Processing: A Status Report. Centre for Sustainable Resource Processing (CSRP), Perth, Western Australia (2005)

Chertow, M.R.: Industrial symbiosis: literature and taxonomy. Annual Review of Energy and the Environment 25, 313-337 (2000)

Chertow, M.R.: "Uncovering” industrial symbiosis. Journal of Industrial Ecology 11(1), 11-30 (2007) 
Chertow, M., Miyata, Y.: Assessing collective firm behavior: comparing Indus-trial Symbiosis with possible alternatives for individual companies in Oahu, HI. Bus. Strat. Env. 20, 266$280(2011)$

European Union (EU), Resource Efficiency Flagship Initiative (2011), http: / / ec.europa.eu/europe2020/europe-2020-in-a-nutshell/ flagship-initiatives/index_en.htm

Frosch, D., Gallopoulos, N.: Strategies for manufacturing. Scientific American 261(3), 94-102 (1989)

Golev, A., Corder, G.D.: Developing a classification system for regional resource synergies. Minerals Engineering 29, 58-64 (2012)

The Interagency Workgroup on Industrial Ecology, Material and Energy Flows 1998. Materials, Washington, DC (1998)

Korhonen, J.: Regional industrial ecology: examples from regional economic systems of forest industry and energy supply in Finland. Journal of Environmental Management 63, 367-375 (2001)

Lombardi, D.R., Laybourn, P.: Redefining Industrial Symbiosis - Crossing AcademicPractitioner Boundaries. Journal of Industrial Ecology 16(1), 28-37 (2012)

McCann, R.: AES Hawaii. Interviews 22 (July 27-October 14, 2005)

Mirata, M., Emtairah, T.: Industrial symbiosis networks and the contribution to environmental innovation: the case of the Landskrona industrial symbiosis programme. Journal of Cleaner Production 13(10-11), 993-1002 (2005)

OECD, Project on Green Growth and Eco-Innovation, OECD (2010)

Research Triangle Institute, Eco-Industrial Parks: A case study and analysis of economic, environmental, technical, and regulatory Issues (1996), http: / / www.rti.org/pubs / case-study.pdf

Storper, M., Harrison, B.: Flessibilità, gerarchie e sviluppo regionale: la ristrutturazione organizzativa dei sistemi produttivi e le nuove forme di governance. In: Belussi, F. (ed.) Nuovi Modelli D’impresa, Gerarchie Organizzative e Impresa Rete, pp. 209-237. Angeli, Milano (1992)

United Nations Environment Programme (UNEP), Global Green New Deal. Policy Brief (2009), http: / / www . unep.org/pdf/GGND_Final_Report.pdf

Van Beers, D., Corder, G., Bossilkov, A., Van Berkel, R.: Industrial Symbiosis in the Australian minerals industry - The cases of Kwinana and Gladstone. Journal of Industrial Ecology 11(1), 55-72 (2007)

Wolf, A.: Industrial Symbiosis in the Swedish Forest Industry. Ph.D. thesis, Linköping University, Linköping (2007)

Yang, S., Feng, N.: A case study of industrial symbiosis: Nanning Sugar Co., Ltd. in China. Resources, Conservation and Recycling 52, 813-820 (2008) 